$8 / 26 / 98$

\title{
CONTROL OF NONLINEAR UNDERACTUATED SYSTEMS
}

\author{
DAVID AUCKLY \\ Department of Mathematics, \\ Kansas State University, Manhattan, KS 66506-2602, USA \\ email: dav@math.ksu.edu \\ LEV KAPITANSKI \\ Department of Mathematics, \\ Kansas State University, Manhattan, KS 66506-2602, USA \\ email: levkapit@math.ksu.edu \\ WARREN White \\ Department of Mechanical and Nuclear Engineering \\ Kansas State University, Manhattan, KS 66506-5106, USA \\ email:wnw@ksu.edu
}

\begin{abstract}
In this paper we introduce a new method to design control laws for non-linear underactuated systems. Our method will often work in situations where standard control techniques fail. Even when standard techniques apply, we believe that our approach will achieve better performance. This is because we produce an infinite dimensional family of control laws, whereas most control techniques only produce a finite dimensional family of control laws. We will describe the problem and our solution invariantly, using differential geometry and in local coordinates. We hope that this paper will be useful for both mathematicians and engineers. We include an abstract example of a system which is open-loop unstable and cannot be stabilized using any linear control law, and demonstrate that our method produces a stabilizing control law. We also apply our method to the inverted pendulum cart to compare it with some standard control techniques.

An important problem in control theory is how to modify a system of ordinary differential equations so that solutions to the equations satisfy some property. In order to
\end{abstract}


describe the class of differential equations that we consider, let $Q$ denote the configuration space. The configuration space is a finite dimensional manifold that represents, for example, every possible position of a mechanical system. Let $g \in \Gamma\left(T^{*} Q \otimes T^{*} Q\right)$ be a metric. This is the mass matrix in a mechanical system. Let $c, f: T Q \rightarrow T Q$ be fiber-preserving maps. These maps are not assumed to be linear, but we do assume that $c$ is odd, i.e., $c(-X)=-c(X)$. In a mechanical system $c$ will represent the inherent dissipation and $f$ will represent the input forces. Finally, let $V: Q \rightarrow \mathbb{R}$. This will represent the potential energy of a mechanical system. The differential equation that we consider in this paper is

$$
\nabla_{\dot{\gamma}} \dot{\gamma}+c(\dot{\gamma})+\operatorname{grad}_{\gamma} V=f(\dot{\gamma})
$$

Let $P \in \Gamma\left(T^{*} Q \otimes T Q\right)$ be a $g$-orthogonal projection. We consider the situation where a constaint $P(f)=0$ is imposed. A system is called underactuated if $P \neq 0$. A mechanical system is underactuated if we require that control forces are zero in certain directions. Many problems in chemical, electrical and mechanical engineering may be formulated as follows: find a function $f$ with $P(f)=0$ so that solutions to Equation (1) have some specified property. For example, solutions with initial conditions in some region will remain close to some path, or as a different example, some point in $T Q$ will be an asymptotically stable equilibrium.

In local coordinates $\underline{x}=\left(x^{1}, \ldots, x^{n}\right)$ Equation $(1)$ reads

$$
\ddot{x}^{k}+\Gamma_{i j}^{k}(\underline{x}) \dot{x}^{i} \dot{x}^{j}+c^{k}(\underline{x}, \underline{\dot{x}})+g^{i k} \frac{\partial V}{\partial x^{i}}=f^{k}(\underline{x}, \underline{\dot{x}})
$$

where $\Gamma_{i j}^{k}(\underline{x})$ are the Christoffel symbols (of the Levi-Civita connection) associated to the metric $g$, [Hicks, 1965], and $g^{i k}$ is the inverse matrix to $g_{k j}$. In Equation (2) and throughout the rest of this paper we are using the summation convention, that repeated indices are summed from 1 to $n$. As we have mentioned, we assume that $c^{k}(\underline{x},-\underline{\dot{x}})=$ $-c^{k}(\underline{x}, \underline{\dot{x}})$ and the projection of $f^{k}$ to some specified set of directions must be zero.

The main question addressed in this paper is how to find a function, $f$, with $P(f)=0$, so that solutions to Equation (1) satisfy some preassigned conditions. Our approach to this question is to find functions $\widehat{g}, \widehat{c}, \widehat{V}$ and $f$ so that solutions to Equation (1) are automatically solutions to

$$
\widehat{\nabla}_{\dot{\gamma}} \dot{\gamma}+\widehat{c}(\dot{\gamma})+\widehat{\operatorname{grad}}_{\gamma} \widehat{V}=0
$$


This will clearly be the case if

$$
f(X) \equiv \nabla_{X} X-\widehat{\nabla}_{X} X+\operatorname{grad}_{\gamma} V-\widehat{\operatorname{grad}}_{\gamma} \widehat{V}+c(X)-\widehat{c}(X),
$$

for every vector field $X$. The condition $P(f)=0$ then becomes a system of nonlinear partial differential equations for $\widehat{g}, \widehat{c}$, and $\widehat{V}$. Notice that constant multiples of $g, c$, and $V$ satisfy $P(f)=0$ even when $P$ has full rank. Thus, one would expect many solutions when $P$ does not have full rank. Separating $P(f)=0$ into terms which are quadratic in the velocity, independent of the velocity or odd functions of the velocity gives

$$
\begin{gathered}
P\left(\nabla_{X} X-\widehat{\nabla}_{X} X\right)=0, \\
P\left(\operatorname{grad}_{\gamma} V-\widehat{\operatorname{grad}}_{\gamma} \widehat{V}\right)=0, \\
P(c(X)-\widehat{c}(X))=0 .
\end{gathered}
$$

We will look for solutions to these matching equations with $\widehat{g}$ non-degenerate so that $g(X, Y)=\widehat{g}(\lambda X, Y)$ with $\lambda \in \Gamma\left(T^{*} Q \otimes T Q\right)$. It is clear that $\lambda$ has to be $g$ self-adjoint, i.e., $g(\lambda X, Y)=g(X, \lambda Y)$. We will derive a linear system of partial differential equations for $\lambda$ which must be satisfied if $\widehat{g}$ is to solve Equation (4.1).

To derive the equations for $\lambda$ we will use the relation between the connection, Lie bracket (commutator) and metric. In fact, we only derive equations for $\left.\lambda\right|_{\operatorname{Im} P}$.

It is known, [Hicks], that the covariant derivative $\nabla$ compatible with the metric $g$ is determined uniquely as a bilinear operator which associates to any pair of vectors $X$ and $Y$ a third vector $\nabla_{X} Y$ so that the following equations are satisfied:

$$
\begin{gathered}
X(g(Y, Z))=g\left(\nabla_{X} Y, Z\right)+g\left(Y, \nabla_{X} Z\right), \\
\nabla_{X} Y-\nabla_{Y} X=[X, Y],
\end{gathered}
$$

where $[X, Y]$ is the Lie bracket (commutator) of $X$ and $Y$. Using the above properties of the covariant derivative we get:

$$
\begin{aligned}
2 g\left(\nabla_{X} Y, Z\right)= & X g(Y, Z)+Y g(Z, X)-Z g(X, Y) \\
& +g([X, Y], Z)+g([Z, X], Y)-g([Y, Z], X) .
\end{aligned}
$$

This may be solved for $\nabla_{X} Y$ since $g$ is non-degenerate. 
Proposition 1. If $g=\widehat{g} \lambda$ and $\widehat{g}$ satisfies the matching condition $P\left(\nabla_{X} X-\widehat{\nabla}_{X} X\right)=0$, then $\lambda$ satisfies:

$$
\left.\nabla g \lambda\right|_{\operatorname{Im} P \otimes 2}=0
$$

or, equivalently,

$$
g\left(\nabla_{Z} \lambda P X, P X\right)-g\left(\lambda P X, \nabla_{Z} P X\right)=0 .
$$

Proof. We begin by polarizing the matching equation

$$
\begin{aligned}
P\left(\nabla_{X} Y-\hat{\nabla}_{X} Y\right)= & \frac{1}{2} P\left(\nabla_{X} Y+\nabla_{Y} X-\widehat{\nabla}_{X} Y-\widehat{\nabla}_{Y} X\right) \\
=\frac{1}{2}\left[P\left(\nabla_{X+Y}(X+Y)-\widehat{\nabla}_{X+Y}(X+Y)\right)\right. & \left.\quad-P\left(\nabla_{X} X-\widehat{\nabla}_{X} X\right)-P\left(\nabla_{Y} Y-\widehat{\nabla}_{Y} Y\right)\right] \\
= & 0 .
\end{aligned}
$$

The first line is true because the covariant derivatives are torsion free (Equation (6)). The second line is true because the covariant derivatives are bilinear (Equation (7)). Now,

$$
\begin{aligned}
0= & 2 g\left(P\left(\nabla_{\lambda P X} Z-\widehat{\nabla}_{\lambda P X} Z\right), X\right) \\
= & 2 g\left(\nabla_{\lambda P X} Z-\widehat{\nabla}_{\lambda P X} Z, P X\right) \\
= & 2 g\left(\nabla_{\lambda P X} Z, P X\right)-2 \widehat{g}\left(\widehat{\nabla}_{\lambda P X} Z, \lambda P X\right) \\
= & \lambda P X g(Z, P X)+Z g(P X, \lambda P X)-P X g(\lambda P X, Z) \\
& \quad+g([\lambda P X, Z], P X)+g([P X, \lambda P X], Z)-g([Z, P X], \lambda P X) \\
& \quad-\lambda P X \widehat{g}(Z, \lambda P X)-Z \widehat{g}(\lambda P X, \lambda P X)+\lambda P X \widehat{g}(\lambda P X, Z) \\
& \quad-\widehat{g}([\lambda P X, Z], \lambda P X)-\widehat{g}([\lambda P X, \lambda P X], Z)+\widehat{g}([Z, \lambda P X], \lambda P X) \\
=\lambda P X g(Z, P X)-P X g(\lambda P X, Z)+g([P X, \lambda P X], Z) & \quad-g([Z, P X], \lambda P X)+g([Z, \lambda P X], P X) \\
= & g\left(\nabla_{\lambda P X} Z, P X\right)+g\left(Z, \nabla_{\lambda P X} P X\right) \\
& \quad-g\left(\lambda P X, \nabla_{P X} Z\right)-g\left(Z, \nabla_{P X} \lambda P X\right) \\
& +g([Z, \lambda P X], P X)-g([P X, \lambda P X], Z)-g([Z, P X], \lambda P X) \\
= & g\left(\nabla_{Z}(\lambda P X), P X\right)-g\left(\nabla_{Z} P X, \lambda P X\right) \\
= & g\left(\left(\nabla_{Z} \lambda\right)(P X),(P X)\right) \\
= & \left(\nabla_{Z} g \lambda\right)(P X, P X) .
\end{aligned}
$$


The covariant derivative is extended to all tensors by requiring every reasonable product rule to hold. For example, $Z(\lambda(X))=\nabla_{Z}(\lambda(X))=\left(\nabla_{Z} \lambda\right)(X)+\lambda\left(\nabla_{Z} X\right)$ and $X(g(Y, Z))=\nabla_{X}(g(Y, Z))=\left(\nabla_{X} g\right)(Y, Z)+g\left(\nabla_{X} Y, Z\right)+g\left(Y, \nabla_{X} Z\right)$. We used these two product rules in the last two lines and Equations (5), (6) and (7) in the previous lines. $\left.\lambda\right|_{\operatorname{Im} P} \cdot$

Now that we have equations for $\left.\lambda\right|_{\operatorname{Im} P}$, we will derive equations for $\widehat{g}$ in terms of

Proposition 2. If $g=\widehat{g} \lambda$, then

$$
\lambda P X \widehat{g}(Z, Z)+2 \widehat{g}([Z, \lambda P X], Z)=2 Z g(P X, Z)-2 g\left(P X, \nabla_{Z} Z\right)
$$

Proof.

$$
\begin{aligned}
\lambda P X & \widehat{g}(Z, Z)+2 \widehat{g}([Z, \lambda P X], Z) \\
& =2 \widehat{g}\left(\widehat{\nabla}_{\lambda P X} Z, Z\right)+2 \widehat{g}([Z, \lambda P X], Z) \\
& =2 \widehat{g}\left(\widehat{\nabla}_{Z} \lambda P X, Z\right) \\
& =2 Z g(P X, Z)-2 g\left(P X, \nabla_{Z} Z\right) .
\end{aligned}
$$

The matching equation for the potential energy may be expressed as a linear partial differential equation using $\lambda$.

Proposition 3. If the matching Equations (4) are satisfied and $g=\widehat{g} \lambda$, then

$$
\lambda P X(\widehat{V})=P X(V) .
$$

Proof. $\quad \lambda P X(\widehat{V})=(d \widehat{V})(\lambda P X)=\widehat{g}\left(\widehat{\operatorname{grad}}_{\gamma} \widehat{V}, \lambda P X\right)=g\left(\widehat{\operatorname{grad}}_{\gamma} \widehat{V}, P X\right)$

$=g\left(P \widehat{\operatorname{grad}}_{\gamma} \widehat{V}, X\right)=g\left(P \operatorname{grad}_{\gamma} V, X\right)=P X(V)$.

By solving the matching equations, we find every control law that will result in dynamical behavior equivalent to a system of the form:

$$
\widehat{\nabla}_{\dot{\gamma}} \dot{\gamma}+\widehat{c}(\dot{\gamma})+\widehat{\operatorname{grad}}_{\gamma} \widehat{V}=0
$$

This is a very large collection of vector fields on $T Q$. Thus far, we have shown that every solution to the matching equations may be found by first solving one system of 
linear partial differential equations and then solving a different system of linear partial differential equations.

The previous three propositions suggest a natural approach for solving the matching equations. First, solve Equation (9) for $\lambda P X$, then solve Equation (10) for $\widehat{g}$, and Equation (11) for $\widehat{V}$. Finally solve the algebraic Equation (4.3) for $\widehat{c}$. Every solution to the matching equations can be found in this way. What is not clear is whether every set of functions generated in this way is a solution to the matching equations. The problem is that once we have a solution to the $\lambda$-equation, (Equation (9)), and then a solution to Equation (10), it is not clear that $\lambda$ can be extended from $\operatorname{Im} P$ so that the condition $g=\widehat{g} \lambda$ holds. We will address this question for general $P$ in a future paper. We will next show that $g=\widehat{g} \lambda$ holds if it is true at the initial conditions, provided that the rank of $P$ is one.

Proposition 4. Let $X$ be a non-zero vector field in an open set $U$, generating $I m P$, and let $\lambda P X$ be a non-vanishing solution of the $\lambda$ Equation (9) in $U$. Let $\Sigma$ be a codimension one non-characteristic hypersurface in $U$. Assume that $\widehat{g}$ is defined, non-degenerate, and satisfies $\widehat{g} \lambda P X=g P X$ on $\Sigma$, and $\widehat{V}$ is defined on $\Sigma$. Then there is a unique solution to the matching Equations (4.1) and (4.2), and a unique extension of $\lambda$ away from Im $P$ in a neighborhood of $\Sigma$. Furthermore, $\widehat{g} \lambda=g$ everywhere in the neighborhood.

Proof. Notice that $\Sigma$ is non-characteristic for the $\widehat{g}$-Equation (10) if it is non-characteristic for the $\widehat{V}$-Equation (11), and visa versa. The functions $\widehat{g}$ and $\widehat{V}$ may be found in a neighborhood of $\Sigma$ using the method of characteristics. The main content of this proposition is that $g P X=\widehat{g} \lambda P X$ everywhere in the neighborhood. After polarization, the $\widehat{g}$-Equation (10) reads:

$$
\begin{aligned}
\lambda P X & \widehat{g}(\lambda P X, Z)+\widehat{g}(\lambda P X,[Z, \lambda P X]) \\
& =Z g(\lambda P X, P X)+\lambda P X g(P X, Z)-g\left(\nabla_{Z} \lambda P X, P X\right)-g\left(\nabla_{\lambda P X} Z, P X\right) \\
& =\lambda P X g(P X, Z)+g(P X,[Z, \lambda P X])+g\left(\lambda P X, \nabla_{Z} P X\right)-g\left(\nabla_{Z} \lambda P X, P X\right) .
\end{aligned}
$$

This last line follows after applying the product rule to the first term of the previous line and the torsion-free condition (6) to the last term of the previous line. In view of the $\lambda$-Equation (9), it is clear that $\widehat{g} \lambda P X=g P X$ is a solution of this equation. This solution is unique in a neighborhood of $\Sigma$. Finally, since $\widehat{g}$ is non-degenerate on $\Sigma$, it is non-degenerate in a neighborhood of $\Sigma$, so we may extend $\lambda$ to the full tangent space by $\lambda=(\widehat{g})^{-1} g$. 


\section{EXAMPLE}

In order to demonstrate and clarify our method, we will apply it to an inverted pendulum cart as an example.

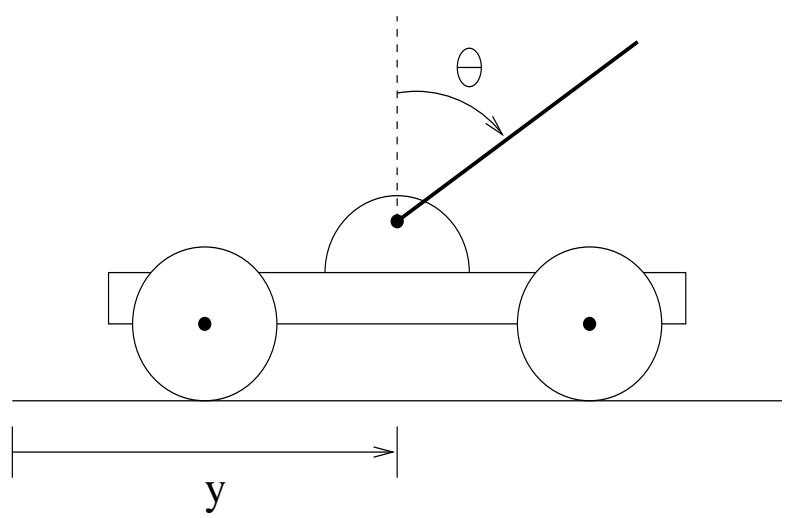

Figure 1 
The (mass) metric for the cart system depicted in Figure 1 is:

$$
g=(M+m) d y^{2}+2 m \ell \cos (\theta) d \theta d y+\left(m \ell^{2}+I\right) d \theta^{2},
$$

and the potential energy is:

$$
V=m \mathrm{~g} \ell \cos (\theta) .
$$

Here

$M$ is the mass of the base of the cart,

$m$ is the mass of the pendulum,

$\ell \quad$ is the length from hinge to the center of mass of the pendulum,

$I \quad$ is the moment of inertial about the center of mass, and

$\mathrm{g}$ is the acceleration due to gravity.

By a change of length, time and mass scales, the metric and the potential energy may be transformed into

$$
g=d x^{2}+2 b \cos (\theta) d \theta d x+d \theta^{2}, \quad V=\cos (\theta),
$$

where $b=m \ell(M+m)^{-\frac{1}{2}}\left(m \ell^{2}+I\right)^{-\frac{1}{2}} \in(0,1)$ is a dimensionless parameter.

The problem is to find the force applied to the base of the cart as a function of the state of the cart so that the origin $(\theta=0, x=0)$ will be an asymptotically stable equilibrium of the resulting dynamical system.

In this application, we may only directly apply force in the $x$ direction, so $P$ is projection onto the $\frac{\partial}{\partial \theta}$ direction, i.e.,

$$
P=(b \cos (\theta) d x+d \theta) \otimes \frac{\partial}{\partial \theta} .
$$

Projecting system (1) onto the coordinate directions gives:

$$
\begin{aligned}
P\left(\nabla_{\dot{\gamma}} \dot{\gamma}+c(\dot{\gamma})+\operatorname{grad}_{\gamma} V\right) & =0, \\
g\left(\frac{\partial}{\partial x}, \nabla_{\dot{\gamma}} \dot{\gamma}+c(\dot{\gamma})+\operatorname{grad}_{\gamma} V\right) & =u \equiv g\left(\frac{\partial}{\partial x}, f\right) .
\end{aligned}
$$

We will assume that there is no inherent dissapation: $c \equiv 0$. Then, in local coordinates, the equations read:

$$
\begin{aligned}
\ddot{\theta}+b \cos (\theta) \ddot{x}-\sin (\theta) & =0 \\
b \cos (\theta) \ddot{\theta}+\ddot{x}-b \sin (\theta) \dot{\theta}^{2} & =u .
\end{aligned}
$$


Equation (3) expresses our solution to this problem in terms of functions, $\widehat{g}_{i j}$, $\widehat{V}$ and $\widehat{c}_{i}, i, j=1$ or 2 , which satisfy the Equation (4). In local coordinates, these matching equations read:

$$
\begin{gathered}
\left(\widehat{g}_{22}-b \cos (\theta) \widehat{g}_{12}\right) \frac{\partial \widehat{g}_{11}}{\partial \theta}+\left(b \cos (\theta) \widehat{g}_{11}-\widehat{g}_{12}\right)\left[2 \frac{\partial \widehat{g}_{12}}{\partial \theta}-\frac{\partial \widehat{g}_{11}}{\partial x}\right]=0 \\
\left(\widehat{g}_{22}-b \cos (\theta) \widehat{g}_{12}\right)\left[2 \frac{\partial \widehat{g}_{12}}{\partial x}-\frac{\partial \widehat{g}_{22}}{\partial \theta}\right]+\left(b \cos (\theta) \widehat{g}_{11}-\widehat{g}_{12}\right) \frac{\partial \widehat{g}_{22}}{\partial x}=0 \\
\left(\widehat{g}_{22}-b \cos (\theta) \widehat{g}_{12}\right) \frac{\partial \widehat{g}_{11}}{\partial x}+\left(b \cos (\theta) \widehat{g}_{11}-\widehat{g}_{12}\right) \frac{\partial \widehat{g}_{22}}{\partial \theta}=0 \\
\left(\widehat{g}_{22}-b \cos (\theta) \widehat{g}_{12}\right) \frac{\partial \widehat{V}}{\partial \theta}+\left(b \cos (\theta) \widehat{g}_{11}-\widehat{g}_{12}\right) \frac{\partial \widehat{V}}{\partial x}=-\sin (\theta)\left(\widehat{g}_{11} \widehat{g}_{22}-\left(\widehat{g}_{12}\right)^{2}\right)
\end{gathered}
$$

and

$$
\widehat{c}_{1}+b \cos (\theta) \widehat{c}_{2}=0
$$

We have slightly simplified these equations by multiplying by the determinant of $\widehat{g}$. (The terms on the right hand side of each equation would be non-zero if the cart was on a hill, and the hinge was rusty. Therefore, multiplying by the determinant of $\widehat{g}$ would not be a great simplification.) These equations are messy, but the same functions may be found by solving the equations in Propositions 1, 2, and 3 without ever writing out the matching equations. Take $X=\frac{\partial}{\partial \theta}$ in Proposition 1 (so $P X=\frac{\partial}{\partial \theta}$ ) and let

$$
\lambda\left(\frac{\partial}{\partial \theta}\right)=\sigma \frac{\partial}{\partial \theta}+\mu \frac{\partial}{\partial x}
$$

Using the bilinearity of the covariant derivative and the product rule, the $\lambda$-Equation (9) becomes:

$$
g\left(\frac{\partial}{\partial \theta}, \frac{\partial}{\partial \theta}\right) \frac{\partial \sigma}{\partial \theta}+g\left(\frac{\partial}{\partial x}, \frac{\partial}{\partial \theta}\right) \frac{\partial \mu}{\partial \theta}+g\left(\nabla_{\frac{\partial}{\partial \theta}} \frac{\partial}{\partial x}, \frac{\partial}{\partial \theta}\right) \mu-g\left(\frac{\partial}{\partial x}, \nabla_{\frac{\partial}{\partial \theta}} \frac{\partial}{\partial \theta}\right) \mu=0
$$

and

$$
g\left(\frac{\partial}{\partial \theta}, \frac{\partial}{\partial \theta}\right) \frac{\partial \sigma}{\partial x}+g\left(\frac{\partial}{\partial x}, \frac{\partial}{\partial \theta}\right) \frac{\partial \mu}{\partial x}+g\left(\nabla_{\frac{\partial}{\partial x}} \frac{\partial}{\partial x}, \frac{\partial}{\partial \theta}\right) \mu-g\left(\frac{\partial}{\partial x}, \nabla_{\frac{\partial}{\partial x}} \frac{\partial}{\partial \theta}\right) \mu=0
$$

Using Equation (7), it follows that:

$$
\begin{aligned}
\frac{\partial \sigma}{\partial \theta}+b \cos (\theta) \frac{\partial \mu}{\partial \theta}+b \sin (\theta) \mu & =0 \\
\frac{\partial \sigma}{\partial x}+b \cos (\theta) \frac{\partial \mu}{\partial x} & =0 . \\
9 &
\end{aligned}
$$


By the Frobenius Theorem, or equivalently checking that mixed derivatives are equal, we see that this system has a solution if and only if $b \sin (\theta) \frac{\partial \mu}{\partial x}=0$. The second equation then implies that $\sigma$ only depends on $\theta$, so the first equation reduces to an ordinary differential equation. The general solution to this system of equations is, therefore,

$$
\sigma=\sigma(\theta), \quad \mu=\mu_{0} \cos (\theta)-\frac{1}{b} \cos (\theta) \int \frac{\partial \sigma}{\partial \theta} \sec ^{2} \theta d \theta
$$

For the rest of this example, we will use the particular solution:

$$
\sigma=\sigma_{0}, \quad \mu=\mu_{0} \cos (\theta)
$$

It is easiest to find $\widehat{g}$ using the basis $\frac{\partial}{\partial \theta}, \lambda\left(\frac{\partial}{\partial \theta}\right)$. By the relation $\widehat{g} \lambda=g$, we already know $\widehat{g}\left(\lambda\left(\frac{\partial}{\partial \theta}\right), \frac{\partial}{\partial \theta}\right)$ and $\widehat{g}\left(\lambda\left(\frac{\partial}{\partial \theta}\right) \lambda\left(\frac{\partial}{\partial \theta}\right)\right)$, so we only need to find $\widehat{g}\left(\frac{\partial}{\partial \theta}, \frac{\partial}{\partial \theta}\right)$. The Lie bracket,

$$
\left[\frac{\partial}{\partial \theta}, \lambda\left(\frac{\partial}{\partial \theta}\right)\right]=-\mu_{0} \sin (\theta) \frac{\partial}{\partial x}=\sigma_{0} \tan (\theta) \frac{\partial}{\partial \theta}-\tan (\theta) \lambda\left(\frac{\partial}{\partial \theta}\right)
$$

may be plugged into the $\widehat{g}$-Equation (10) to get:

$$
\sigma_{0} \frac{\partial \widehat{g}_{11}}{\partial \theta}+\mu_{0} \cos (\theta) \frac{\partial \widehat{g}_{11}}{\partial x}+2 \sigma_{0} \tan (\theta) \widehat{g}_{11}-2 \tan (\theta)=0
$$

The surface $\theta=0$ is non-characteristic, so the above equation may be solved with initial data $\widehat{g}_{11}(0, x)=h(x)$.

The flow of the vector field $\lambda P X$ is given by:

$$
\dot{\theta}=\sigma_{0}, \quad \dot{x}=\mu_{0} \cos (\theta)
$$

or

$$
\theta=\sigma_{0} t, \quad x=a+\frac{\mu_{0}}{\sigma_{0}} \sin \left(\sigma_{0} t\right),
$$

using $\theta=0$ at $t=0$. With this flow, the $\widehat{g}$ equation becomes:

$$
\frac{d \widehat{g}_{11}}{d t}+2 \sigma_{0} \tan \left(\sigma_{0} t\right) \widehat{g}_{11}-2 \tan \left(\sigma_{0} t\right)=0
$$

So

$$
\widehat{g}_{11}=\frac{1}{\sigma_{0}}+\left(h(a)-\frac{1}{\sigma_{0}}\right) \cos ^{2}\left(\sigma_{0} t\right)=\frac{1}{\sigma_{0}}+\left(h\left(x-\frac{\mu_{0}}{\sigma_{0}} \sin (\theta)\right)-\frac{1}{\sigma_{0}}\right) \cos ^{2}(\theta) .
$$


Again, we only take a particular solution:

$$
\widehat{g}_{11}=\frac{1}{\sigma_{0}}+r \cos ^{2}(\theta)
$$

where $r$ is a constant. The equations

$$
b \cos (\theta)=g\left(\frac{\partial}{\partial \theta}, \frac{\partial}{\partial x}\right)=\widehat{g}\left(\lambda\left(\frac{\partial}{\partial \theta}\right), \frac{\partial}{\partial x}\right)=\sigma_{0} \widehat{g}_{12}+\mu_{0} \cos (\theta) \widehat{g}_{22}
$$

and

$$
1=g\left(\frac{\partial}{\partial \theta}, \frac{\partial}{\partial \theta}\right)=\widehat{g}\left(\lambda\left(\frac{\partial}{\partial \theta}\right), \frac{\partial}{\partial \theta}\right)=\sigma_{0} \widehat{g}_{11}+\mu_{0} \cos (\theta) \widehat{g}_{12}
$$

may now be solved for the remaining terms of $\widehat{g}$ :

$$
\widehat{g}_{12}=-\frac{\sigma_{0}}{\mu_{0}} r \cos (\theta), \quad \widehat{g}_{22}=\frac{b}{\mu_{0}}+\frac{\sigma_{0}^{2}}{\mu_{0}^{2}} r .
$$

We can compute $\widehat{V}$ in the same way. Using the $\lambda X P$ flow and initial data $\widehat{V}(0, x)=$ $w(x)$, the $\widehat{V}$-Equation (11) may be written as an ordinary differential equation:

$$
\frac{d \widehat{V}}{d t}=-\sin \left(\sigma_{0} t\right)
$$

Thus,

$$
\widehat{V}=\frac{1}{\sigma_{0}}\left(\cos \left(\sigma_{0} t\right)-1\right)+w(a)=\frac{1}{\sigma_{0}}(\cos (\theta)-1)+w\left(x-\frac{\mu_{0}}{\sigma_{0}} \sin \theta\right) .
$$

Finally, solving for $\widehat{c}$, we get:

$$
\widehat{c}(X)=K(\theta, x, \dot{\theta}, \dot{x})\left(b \cos (\theta) \frac{\partial}{\partial \theta}-\frac{\partial}{\partial x}\right)
$$

with an arbitrary function $K$.

The functions $\widehat{g}, \widehat{V}$ and $\widehat{c}$ produce a family of control laws via equation (3). In this example, the control laws depend on four functions, $\sigma_{0}(\theta), h(x), w(x)$, and $K(\theta, x, \dot{\theta}, \dot{x})$, and one constant, $\mu_{0}$. We have already chosen $\sigma_{0}$ and $h$ to be constants. We will address the question of how to choose the unknown functions and parameters in order to best meet specific design criteria in a future paper. For now, we will just pick elementary functions that will insure that $\theta=0, x=0$ is an asymptotically stable equilibrium. If 
$(0,0)$ is an equilibrium, $\widehat{\operatorname{grad}} \widehat{V}(0,0)$ must be zero, so $w^{\prime}(0)$ must be zero. One standard way to insure that $(0,0)$ will be an asymptotically stable equilibrium is to pick positive definite $\widehat{g}, \widehat{V}$ and $\widehat{g} \widehat{c}$, then $\widehat{H}(X) \equiv \frac{1}{2} \widehat{g}(X, X)+\widehat{V}$ will be a Lyapunov function with time rate of change $-\widehat{g}(\widehat{c}(X), X)$. The Hessian of $\widehat{V}$ at $(0,0)$ is

$$
D_{(0,0)}^{2}=\left(\begin{array}{cc}
\frac{\mu_{0}^{2}}{\sigma_{0}^{2}} w^{\prime \prime}(0)-\frac{1}{\sigma_{0}} & -\frac{\mu_{0}}{\sigma_{0}} w^{\prime \prime}(0) \\
-\frac{\mu_{0}}{\sigma_{0}} w^{\prime \prime}(0) & w^{\prime \prime}(0)
\end{array}\right) .
$$

So we should require:

$$
w^{\prime \prime}(0)>0 \quad \text { and } \quad \operatorname{det} D_{(0,0)}^{2} \widehat{V}=-\frac{1}{\sigma_{0}} w^{\prime \prime}(0)>0 .
$$

We will choose $\sigma_{0}<0$, and $w(z)=\frac{1}{2} w_{1} z^{2}$ with $w_{1}>0$.

The mass matrix should also be positive definite. Thus we should have $\widehat{g}_{11}>0$ and $\widehat{g}_{22}>0$ so that $r>0$ and $b \mu_{0}+\sigma_{0}^{2} r>0$. In addition, the determinant of the mass matrix will also be positive:

$$
\frac{b}{\sigma_{0} \mu_{0}}+\frac{b r}{\mu_{0}} \cos ^{2}(\theta)+\frac{\sigma_{0}}{\mu_{0}^{2}} r>0 .
$$

Rearranging and using the fact that $\sigma_{0}<0$ gives:

$$
-\sigma_{0} b \mu_{0} r \cos ^{2}(\theta)>\sigma_{0}^{2} r+b \mu_{0}
$$

So,

$$
\mu_{0}>\frac{\sigma_{0}^{2} r+b \mu_{0}}{-\sigma_{0} b r \cos ^{2}(\theta)}>0
$$

and

$$
\cos ^{2}(\theta)>\frac{\sigma_{0}^{2} r+b \mu_{0}}{-\sigma_{0} b \mu_{0} r}
$$

Assuming $\mu_{0}>0, r>0$ and $\sigma_{0}<0$, the mass matrix will be positive definite provided condition (16) holds. When the mass matrix and potential energy of the model system are positive definite, the point $(0,0)$ will be Lyapunov stable for any positive semi-definite $\widehat{g} \widehat{c}$. In particular, when $K \equiv 0$ (i.e., $\widehat{c} \equiv 0$ ), we will get Lyapunov stability. We call controllers with $\widehat{c} \equiv 0$ conservative controllers. A direct computation shows that:

$$
\begin{aligned}
\widehat{g}(\widehat{c}(X), X) & =K \widehat{g}\left(b \cos (\theta) \frac{\partial}{\partial \theta}-\frac{\partial}{\partial x}, \dot{\theta} \frac{\partial}{\partial \theta}+\dot{x} \frac{\partial}{\partial x}\right) \\
& =K\left[\left(b \cos (\theta) \widehat{g}_{11}-\widehat{g}_{12}\right) \dot{\theta}+\left(b \cos (\theta) \widehat{g}_{12}-\widehat{g}_{22}\right) \dot{x}\right] \\
& =(\operatorname{det} \widehat{g}) \cdot K \cdot\left(\mu_{0} \cos \theta \dot{\theta}-\sigma_{0} \dot{x}\right) .
\end{aligned}
$$


This will be positive semi-definite if $K=\Phi(\theta, x)\left(\mu_{0} \cos \theta \dot{\theta}-\sigma_{0} \dot{x}\right)$, where $\Phi$ is any positive function. (We can do no better because $\lambda P X=\sigma_{0} \frac{\partial}{\partial \theta}+\mu_{0} \cos \theta \frac{\partial}{\partial x}$ will be a zero mode of any admissible $\widehat{c}$.) We will see that this is sufficient to prove that $(0,0)$ is an asymptotically stable equilibrium of the controlled system. Equation (3), equation (7) and our values for $\widehat{g}, \widehat{V}$ and $\widehat{c}$ combine together to give:

$$
\begin{aligned}
u= & g\left(f, \frac{\partial}{\partial x}\right)=\left(b+\frac{r \operatorname{det} g}{\mu_{0} \operatorname{det} \widehat{g}}\right)\left(\cos (\theta) \sin (\theta)-\sin (\theta) \dot{\theta}^{2}\right) \\
& -\frac{w_{1} \operatorname{det} g}{\sigma_{0} \operatorname{det} \widehat{g}}\left(x-\frac{\mu_{0}}{\sigma_{0}} \sin (\theta)\right)+\operatorname{det} g \Phi(\theta, x)\left(\mu_{0} \cos (\theta) \dot{\theta}-\sigma_{0} \dot{x}\right),
\end{aligned}
$$

where

$$
\operatorname{det} g=1-b^{2} \cos ^{2}(\theta), \quad \operatorname{det} \widehat{g}=\frac{b}{\sigma_{0} \mu_{0}}+\frac{b r}{\mu_{0}} \cos ^{2}(\theta)+\frac{\sigma_{0} r}{\mu_{0}^{2}} .
$$

Thus, we have almost proved the following result.

Proposition 5. Let constants $\mu_{0}, \sigma_{0}, r$, and $w_{1}$ satisfy the conditions

$$
\mu_{0}>0, \quad \sigma_{0}<0, \quad w_{1}>0, \quad 1>\frac{\sigma_{0}^{2} r+b \mu_{0}}{-\sigma_{0} b \mu_{0} r} .
$$

Let $\Phi(\theta, x)$ be any strictly positive function.

Then $(0,0)$ is an asymptotically stable equilibrium of the controlled system (14) with the control law defined by (17), (18).

Proof. Define the controlled Hamiltonian $\widehat{H}=\frac{1}{2} \widehat{g}(\dot{\gamma}, \dot{\gamma})+\widehat{V}$. We have shown above that the Hessian of $\widehat{H}$ is positive definite in some neighborhood of $(0,0)$ when conditions (19) hold. The time derivative of $\widehat{H}$ is:

$$
\begin{aligned}
\frac{d}{d t}(\widehat{H}) & =\frac{d}{d t}\left(\frac{1}{2} \widehat{g}(\dot{\gamma}, \dot{\gamma})+\widehat{V}\right) \\
& =\widehat{g}\left(\nabla_{\dot{\gamma}} \dot{\gamma}+\widehat{\operatorname{grad}} \widehat{V}, \dot{\gamma}\right) \\
& =-\widehat{g}(\widehat{c}(\dot{\gamma}), \dot{\gamma}) \\
& =-\operatorname{det} \widehat{g} \Phi(\theta, x)\left(\mu_{0} \cos (\theta) \dot{\theta}-\sigma_{0} \dot{x}\right)^{2}
\end{aligned}
$$

Check that there is no solution to the controlled equations satisfying $\mu_{0} \cos (\theta) \dot{\theta}-\sigma_{0} \dot{x} \equiv 0$. Since $\Phi>0$ by assumption and $\left.\operatorname{det} \widehat{g}\right|_{(\theta, x)}>0$ for all $(\theta, x)$ sufficiently close to $(0,0)$, the Hamiltonian $\widehat{H}$ decreases along the solutions of (17), and, therefore, may serve as a Lypunov function. The (local) asymptotic stability follows from Lyapunov's Theorem. 
Remark 1. It is not possible to construct a control law so that $(0,0)$ is a globally asymptotically stable equilibrium.

Indeed, the solutions of the controlled system for such a control law would produce a continuous function, $F:[0, \infty] \times T Q \rightarrow T Q$ so that $F_{0}$ is the identity map and $F_{\infty}$ is the constant map. In other words, the flow of a vector field with a globally asymptotically stable equilibrium is a contraction. However, for the inverted pendulum cart, $T Q \simeq S^{1} \times \mathbb{R}^{3}$, which is not contractible.

Since the inverted pendulum cart cannot be globally stabilized by a control law, we can only try to maximize the size of the basin of attraction. We will compare a special case of our nonlinear control law with a linear control law that has been implemented on an inverted pendulum cart in our lab. Our cart has

$$
\begin{array}{rlrl}
M & =5.02 \mathrm{Kg} & m & =.454 \mathrm{Kg} \\
\ell & =.425 \mathrm{~m} & I & =.11 \mathrm{Kg} m^{2}
\end{array}
$$

giving $b=.188$. The linear control law that we obtained by placing poles at $-5,-6$, and a double pole at -2 is:

$$
u_{\operatorname{lin}}=1021 \theta+115.8 x+918.5 \dot{\theta}+158.2 \dot{x} .
$$

This is only an approximation of the actual control law that is running in the lab. In the lab we had to model the DC motor, the observer, and use a discrete control law.

Thus far, we have chosen each of the arbitrary functions in our non-linear control law to be a constant, and we took $w(x)=\frac{1}{2} w_{1} x^{2}$, since it has to have positive second derivative. As a rough guess we took $\mu_{0}=10, \sigma_{0}=-.05, r=1000$, and $w_{1}=1.5$. We also set the function $\Phi=1$. These constants satisfy conditions (19). We chose the functions to be constants when possible just to simplify the exposition.

Remark 2. This would not be the best choice for engineering applications. In particular, $-\sigma_{0}$ controls the coefficient of $\dot{x}$ in our control law. In order to stabilize the system after a large angular disturbance, we would like $-\sigma_{0}$ to be small so that the cart will be free to accelerate under the pendulum. On the other hand, $-\sigma_{0}$ needs to be much bigger in order to insure that the time constant is reasonable. We hope to describe some practical 
approches to choosing the arbitrary functions which appear in our family of control laws so that the resulting controler will meet specific design criteria in a future paper.

For a first rough test of our control law, we ran numerical simulations of the system using both our control law and a linear control law with the arbitrary functions and parameters specified above. We used 10000 diferent sets of initial conditions. It appears that the linear controler has a shorter settling time than the nonlinear control law, when it does not blow up. However, the nonlinear control law appears to stabilize the system starting from any of the initial conditions stabilized by the linear control law, and also appears to stabilize the system starting from many initial conditions for which the linear control law produces an unbounded response.
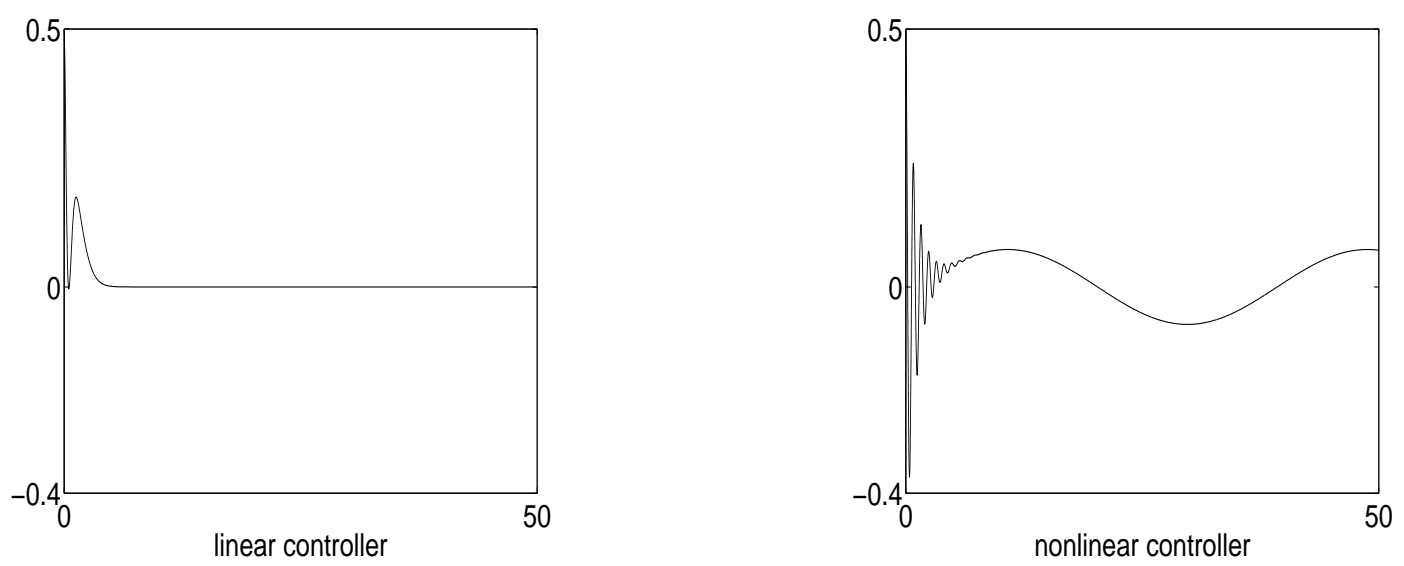

Figure 2

Figure 2 shows graphs of the angular position versus time produced using the linear control law and the nonlinear control law starting from initial conditions: $\theta=.5, \dot{\theta}=$ $-.5, x=0$, and $\dot{x}=0$. The graphs of the other state variables and control input are qualitatively similar. This output is typical of the responce obtained when both control laws appear to stabilize the system. 

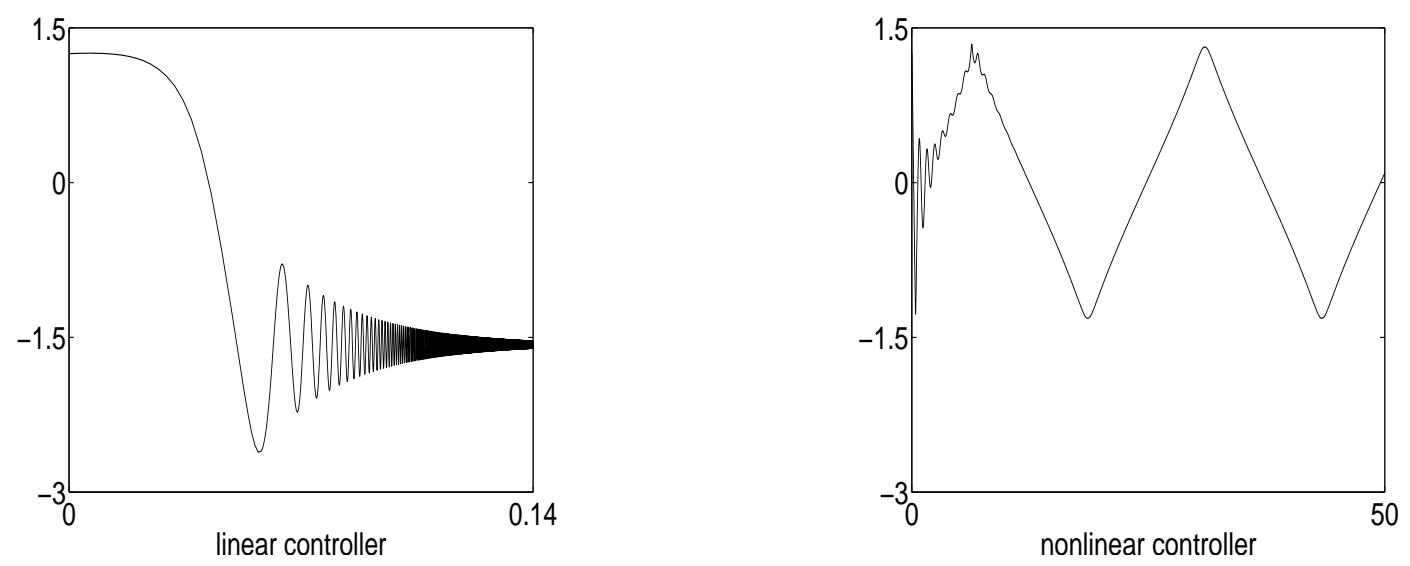

Figure 3

Figure 3 shows graphs of the angular position versus time produced using the linear control law and the nonlinear control law starting from initial conditions: $\theta=1.25$, $\dot{\theta}=1.3, x=0$, and $\dot{x}=0$. For the same initial conditions, Figure 4 shows graphs of the cart position versus time produced using the linear control law and the nonlinear control law.
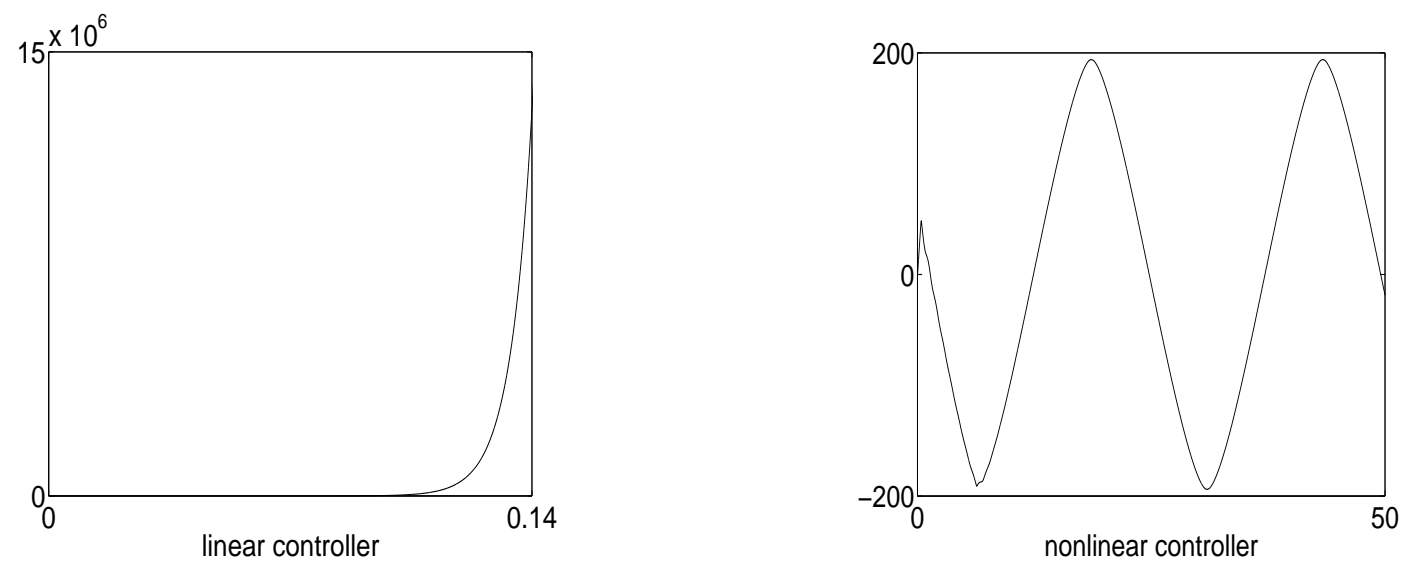

Figure 4

For the linear controller, $x(t)$ increases exponentially. For the nonlinear controller, $x(t)$ stabilizes to 0 , but the system is underdamped, as we discussed previously. The graphs of the other state variables and control input are again qualitatively similar. These graphs are representative for initial conditions for which the linear control blows up, and the nonlinear control stabilizes. All of our numerical simulations were produced using MATLAB 5.1.

Our infinite dimensional family of control laws contains a one dimensional family of 
control laws, which was found previously. Bloch, Leonard and Marsden have developed a method for constructing control laws for mechanical systems with symmetry, [Bloch, Lenard, Marsden, 1997]. Their control law will retain the symmetry, so it cannot produce a truly Lyapunov stable equilibrium. It will only produce a Lyapunov stable equilibrium in shape space. This means that their method will not work for an inverted pendulum cart on a hill, say. If the cart is on level ground, the quantity $\dot{x}+A \cos (\theta) \dot{\theta}$ will be conserved. The quantity $\dot{x}+A \cos (\theta) \dot{\theta}$ is conserved in the closed-loop dynamics of any of the control laws developed by Bloch, Leonard and Marsden. It follows that any solution which passes through a point with $\dot{\theta}=0$ and $\dot{x} \neq 0$ will run off to infinity.

\section{LINEARLY NON-CONTROLLABLE EXAMPLE}

The final example that we will consider is an abstract system which is open-loop unstable with the property that the linearized system cannot be stabilized by any control law. Our method will generate a control law with a globally asymptotically stable equilibrium.

The abstract system is: $g=d x^{2}+d y^{2}, P=d y \otimes \frac{\partial}{\partial y}$ and $V=-\frac{3}{2} x^{4}+45 x^{2} y^{2}+32 x y^{3}$. In coordinates, the control problem reads:

$$
\begin{aligned}
& \ddot{x}-6 x^{3}+90 x y^{2}+32 y^{3}=0 \\
& \ddot{y}+90 x^{2} y+96 x y^{2}=u .
\end{aligned}
$$

This system is, clearly, open-loop $(u=0)$ unstable. For example, $x=\left(\varepsilon^{-1}-\sqrt{3} t\right)^{-1}$, $y=0$ is a solution to the system for any positive $\varepsilon$. The linearized version of the system is: $\ddot{x}=0, \ddot{y}=u$. Clearly, $u$ can have no effect on $x$.

To apply our method to this system, let $\lambda P X=\sigma \frac{\partial}{\partial x}+\mu \frac{\partial}{\partial y}$, then the $\lambda$-Equation (9) reads:

$$
\frac{\partial \sigma}{\partial x}=0, \quad \frac{\partial \sigma}{\partial y}=0 .
$$

Thus, $\sigma$ is any constant and $\mu$ is any function. Pick $\sigma=1$ and $\mu=1$. The $\widehat{g}$-Equation (10) becomes:

$$
\frac{\partial \widehat{g}_{11}}{\partial x}+\frac{\partial \widehat{g}_{11}}{\partial y}=0 .
$$

Pick $\widehat{g}_{11}=2$. The equations $\widehat{g} \lambda=g$ lead to $\widehat{g}_{22}=1$ and $\widehat{g}_{12}=-1$. It is easy to check that the model mass matrix is positive definite. The flow equation for $\widehat{V}$ is

$$
\frac{\partial \widehat{V}}{\partial x}+\frac{\partial \widehat{V}}{\partial y}=-6 x^{3}+90 x y^{2}+32 y^{3}
$$


It is not hard to check that

$$
\widehat{V}=\left(x^{2}-3 x y\right)^{2}+\left(x^{2}-4 x y-2 y^{2}\right)^{2}
$$

is a positive definite solution to this equation. Finally, pick $\widehat{c}=(\dot{y}-\dot{x}) \frac{\partial}{\partial y}$ as a solution to $P(\widehat{c})=P(c)=0$. As before, we show that there is no non-trivial solution to the controlled equations with $\dot{y}-\dot{x} \equiv 0$. Thus, $(0,0)$ is a globally asymptotically stable equilibrium.

\section{REFERENCES}

A. Bloch, N. Lenard, J. Marsden, Stabilization of Mechanical Systems Using Controlled Lagrangians, Proc. 1997 IEEE Conference on Decision and Control (1997), 23562361.

N. Hicks, Notes on Differential Geometry, Van Nostrad, 1965. 